\title{
Predictive factors of submucosal fibrosis before endoscopic submucosal dissection for superficial squamous esophageal neoplasia
}

Cheal Wung Huh', Han Hee Lee², Byung-Wook Kim¹, Joon Sung Kim, Bo-In Lee², Chul-Hyun Lim² and Jiyoung Kim³

\begin{abstract}
Objectives: Endoscopic submucosal dissection (ESD) is an effective treatment modality for superficial squamous esophageal neoplasia (SSEN). However, submucosal fibrosis is an important obstacle to successful ESD. The aim of this study was to determine the ESD outcome in relationship to the degree of submucosal fibrosis of SSEN and to identify factors for predicting submucosal fibrosis.

Methods: We retrospectively analyzed endoscopic and pathologic factors related to submucosal fibrosis in 41 patients with SSEN who underwent ESD. Also, en bloc resection rate, complication rate, and procedure time according to the degree of submucosal fibrosis were evaluated. Masson's trichrome staining was used to evaluate histologic submucosal fibrosis.

Results: A depressed type tumor (vs. nondepressed type tumor, $P=0.002$ ), length of tumor greater than $20 \mathrm{~mm}$ (vs. length of tumor $\leq 20 \mathrm{~mm}, P=0.036$ ), and delayed ESD after initial biopsy (vs. immediate ESD after initial biopsy, $P=$ 0.005 ) were independent factors predictive of submucosal fibrosis. The severity of submucosal fibrosis was significantly associated with a higher complication rate such as bleeding and perforation. Also, as the severity of the submucosal fibrosis increased, the amount of time required for the ESD procedure increased.

Conclusions: Length of tumor greater than $20 \mathrm{~mm}$ and endoscopic depressed type are endoscopic predictive factors of submucosal fibrosis in SSEN. Moreover, to avoid submucosal fibrosis, ESD should be attempted immediately after biopsy for the diagnosis of SSEN.
\end{abstract}

\section{Introduction}

Endoscopic submucosal dissection (ESD) is considered a feasible procedure for superficial squamous esophageal neoplasia $(\mathrm{SSEN})^{1-4}$. Compared with endoscopic mucosal

\section{Correspondence: B.-W. Kim (gastro@catholic.ac.kr)}

${ }^{1}$ Division of Gastroenterology, Department of Internal Medicine, Incheon St. Mary's Hospital, College of Medicine, The Catholic University of Korea, Incheon, Korea

${ }^{2}$ Division of Gastroenterology, Department of Internal Medicine, Seoul St.

Mary's Hospital, College of Medicine, The Catholic University of Korea, Seoul, Korea

Full list of author information is available at the end of the article. resection (EMR), ESD has many advantages such as a higher en bloc resection rate and a lower rate of recurrence $^{5-8}$. However, esophageal ESD is a more difficult procedure to perform than esophageal EMR. Also, esophageal ESD is technically more difficult than gastric ESD. Therefore, esophageal ESD is more likely to lead to a higher frequency of complications (e.g., bleeding and perforation) compared with esophageal EMR or gastric ESD.

Success rate of ESD depends on the expertize of the endoscopist, features of the neoplasia and various applied

\section{() The Author(s) 2018}

(c) (i) Open Access This article is licensed under a Creative Commons Attribution 4.0 International License, which permits use, sharing, adaptation, distribution and reproduction in any medium or format, as long as you give appropriate credit to the original author(s) and the source, provide a link to the Creative Commons license, and indicate if changes were made. The images or other third party material in this article are included in the article's Creative Commons license, unless indicated otherwise in a credit line to the material. If material is not included in the article's Creative Commons license and your intended use is not permitted by statutory regulation or exceeds the permitted use, you will need to obtain permission directly from the copyright holder. To view a copy of this license, visit http://creativecommons.org/licenses/by/4.0/. 
techniques ${ }^{7,9}$. Lesion factors, such as submucosal fibrosis, are important obstacles to success. Previous studies demonstrated that there was significant association between the degree of submucosal fibrosis and the outcome of ESD in gastric and colorectal neoplasia ${ }^{10-12}$. To our knowledge, however, there has been no report on the relationship between the degree of submucosal fibrosis and the outcome of ESD in SSEN. In addition, the prediction of submucosal fibrosis prior to ESD might be helpful for successful procedure. Therefore, we retrospectively investigated the following factors: (1) the association between clinicopathological factors and endoscopic submucosal fibrosis, (2) the relationship between the degree of endoscopic submucsoal fibrosis and the outcome of ESD, (3) the agreement between endoscopically-observed submucsoal fibrosis and pathologically-observed submucosal fibrosis.

\section{Methods Patients}

Between January 2011 and December 2016, a total of 63 patients were diagnosed as esophageal tumors and underwent ESD at Incheon St. Mary's Hospital and at Seoul St. Mary's Hospital, The Catholic University Korea. Twenty two patients with a subepithelial lesion (e.g., gastrointestinal stromal tumor, leiomyoma, granular cell tumor) were excluded and 41 patients were selected for retrospective analysis. The Institutional Review Boards (IRBs) of The Catholic University of Korea approved this study (OC17RESI0131).

\section{ESD procedures}

All ESD procedures were performed by three expert ESD endoscopists (B.W.K., B.I.L., J.S.K). Patients were moderately sedated with midazolam and propofol, while the ESD was performed. A video endoscope with a waterjet function (GIF-HQ290, GIF-Q260J; Olympus, Tokyo, Japan) was used. A disposable distal transparent cap (D-201-11804; Olympus, Tokyo, Japan) was mounted on the tip of the endoscope in all cases. To identify the target lesion, chromoendoscopy with Lugol's solution or narrow band imaging with magnification was used. The area around the lesion was marked with argon plasma coagulation. A mixture of $10 \%$ glycerol solution and diluted epinephrine $(1: 200,000)$ was injected into the submucosal layer under the lesion. Epinephrine $(1: 1000$, total epinephrine $1 \mathrm{mg}$ ) were mixed in a 200-mL container of Glycerol, and $8 \mathrm{~mL}$ of the solution was drawn into $10-\mathrm{mL}$ disposable syringe to use for SSEN. Carbon dioxide was used for the insufflation. The ESD procedure was performed mainly with a dual knife (KD-650Q; Olympus, Tokyo, Japan) or with an IT-knife 2 (KD-610L; Olympus, Tokyo, Japan) or with hook knife (KD-620LR; Olympus, Tokyo, Japan). Hemostatic forceps (Coagrasper, FD-
410LR; Olympus, Tokyo, Japan) with a soft coagulation mode were used to control bleeding during the procedure.

\section{Definitions}

Endoscopically, the degree of submucosal fibrosis was categorized as follows based on the observation at the time of the injection of glycerol mixture (Fig. 1); F0, no fibrosis, which manifests as a transparent layer; F1, mild fibrosis, which appeared as a white web-like structure in the submucosal layer; and F2, severe fibrosis, which appeared as a white muscular structure without a transparent layer in the submucosal layer ${ }^{12}$.

The histological sections were examined by an expert pathologist (J.K.) who was blinded to all clinical information. Masson's trichrome staining was used to evaluate submucosal fibrosis. The intensity of the submucosal fibrosis was evaluated as follows; 0, negative stain, no fibrosis, nearly normal appearance; 1 , weak fibrosis; and 2, dense fibrosis. The extent of fibrosis was calculated by measuring the percentage of the total area stained with Masson's trichrome stain, as follows; $0,0-10 \% ; 1,11-50 \%$; and $2,51-100 \%$. The staining score was finally collected as the sum of the intensity and extent scores. A final score of 0 with fibrosis was considered as no fibrosis (F0), 1 and 2 as mild fibrosis (F1), and 3 and 4 as severe fibrosis (F2), respectively (Fig. 2).

Perforation was defined as a hole which is visible in the esophageal wall where the mediastinal cavity is exposed. Immediate bleeding was defined as spurting or oozing of blood from a vessel during the ESD procedure. Delayed bleeding was defined as bleeding with hematemesis or melena that required endoscopic re-intervention or transfusion after the ESD procedure. The procedure time was defined as the time from the insertion of the endoscope to complete resection of the lesion. The endoscopic gross appearances of SSEN were classified according to the Paris classification as protruding (I), non-protruding and non-excavated (II), or excavated (III) $)^{13}$. Type II lesions were subclassified as slightly elevated (IIa), flat (IIb), or slightly depressed (IIc). Next, all lesions were broadly classified into two groups: depressed type (IIc, III) and nondepressed type (I, IIa, IIb).

\section{Statistical analysis}

Chi-square tests and Fisher's exact tests were used to evaluate associations among the various categorical variables, and $t$-tests were used for non-categorical variables in the intergroup comparisons of clinicopathologic characteristics. A multiple logistic regression analysis was used to determine independent predictors of submucosal fibrosis. In addition, a kappa statistic was used to measure the agreement between the estimates of the endoscopic submucosal fibrosis and histologic submucosal fibrosis. The accepted significance level was a $P$ value less than 


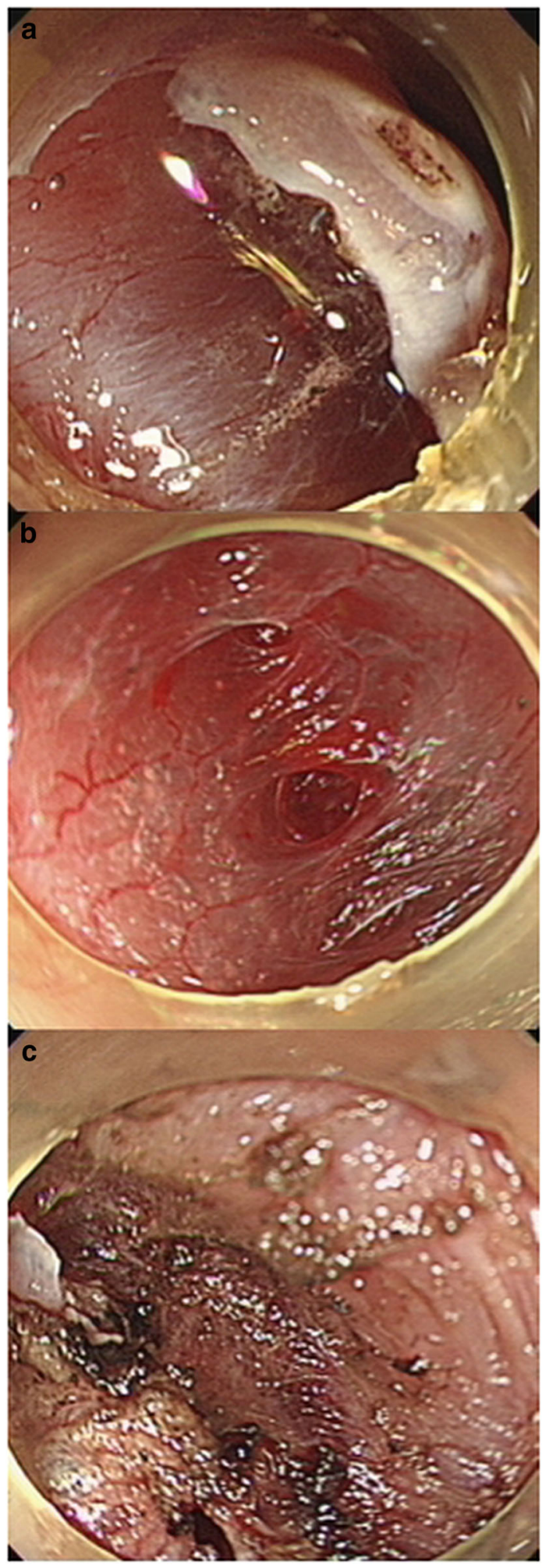

Fig. 1 Degree of fibrosis in the submucosal layers in superficial squamous esophageal neoplasia. a F0, no fibrosis, which manifested as a blue transparent layer. b F1, mild fibrosis, which appears as a white web-like structure in the blue submucosal layer. c F2, severe fibrosis, which appears as a white muscular structure without a blue transparent layer in the submucosal layer

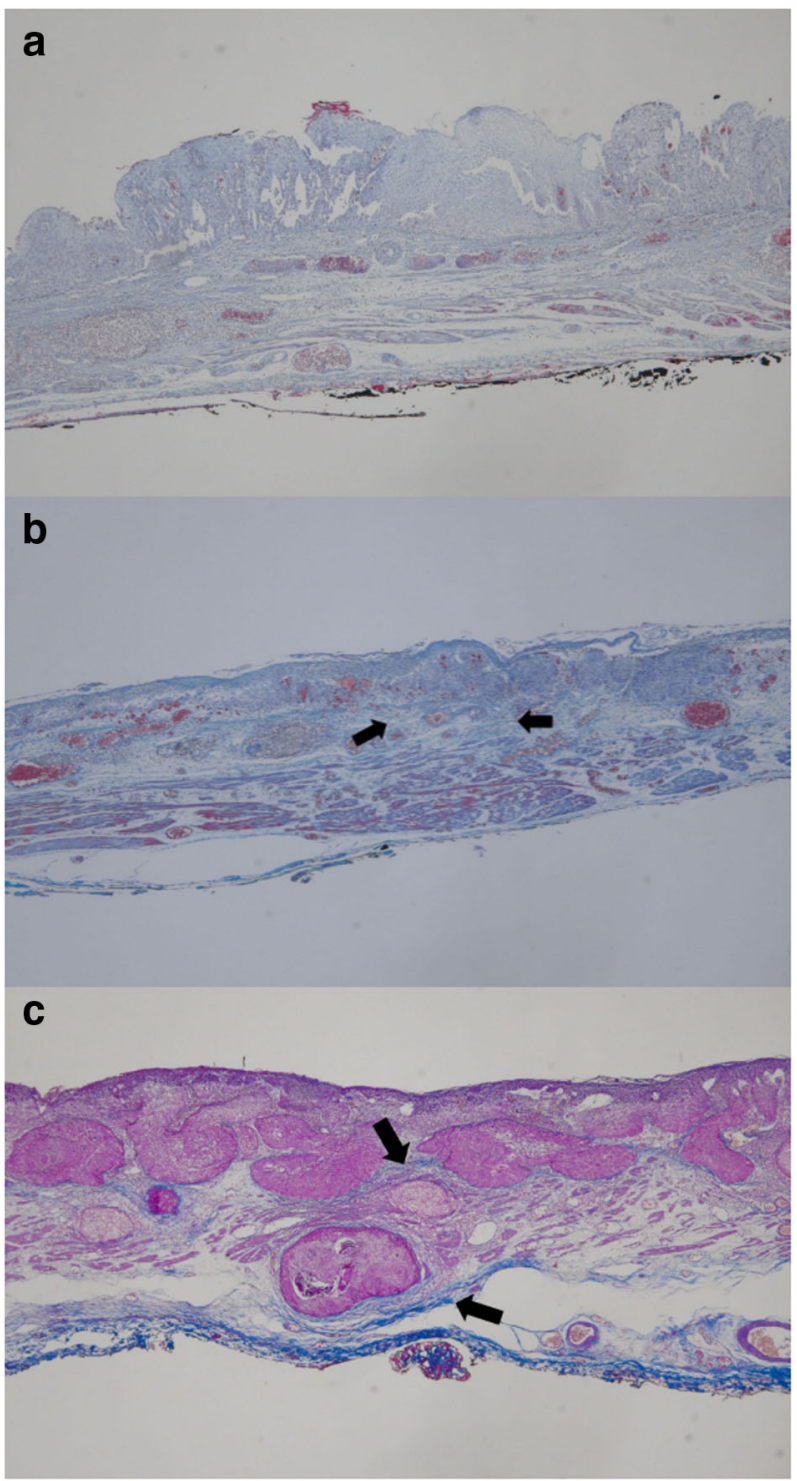

Fig. 2 Histologic assessment of the submucosal fibrosis using Masson's trichrome stain. (Blue: collagen (arrows), Pink: cytoplasm; Brown-dark blue: nuclei) a F0, no fibrosis. (intensity, 0; extent, 0; sum, 0) b F1, mild fibrosis. (intensity, 1; extent, 1; sum 2) c F2, severe fibrosis. (intensity, 2; extent, 2; sum, 4). (Masson's trichrome stain, $\times 40$ )

0.05. All statistical analyses were performed using the SPSS version 18.0 for Windows software (SPSS Inc., Chicago, IL, USA).

\section{Results}

The relationship between clinicopathologic factors and endoscopic submucosal fibrosis

Clinicopathologic factors associated with endoscopic submucosal fibrosis are displayed in Table 1. A univariate analysis demonstrated that endoscopic submucosal fibrosis was significantly associated with length of tumor, endoscopic gross appearance, and timing of ESD from 
Table 1 Univariate analysis of the clinicopathological factors associated with submucosal fibrosis

\begin{tabular}{llll}
\hline Variables & \multicolumn{2}{l}{ Submucosal fibrosis $(n, \%)$} & $P$ \\
\cline { 2 - 3 } vo & \multicolumn{2}{l}{ Yes } \\
\cline { 2 - 4 } No $(N=21)$ & F1 (Mild) & F2 \\
$(n, \%)$ & $(N=15)$ & $\begin{array}{l}(\text { Severe) } \\
(n, \%)\end{array}$ & $(N=5)$ \\
& & $(n, \%)$
\end{tabular}

\begin{tabular}{llll}
\hline Sex & & & \\
Male & $16(80.0)$ & $13(86.7)$ & $3(60.0)$ \\
Female & $4(20.0)$ & $2(13.3)$ & $2(40.0)$ \\
Age (yr) & $63.7 \pm 8.7$ & $65.1 \pm 10.1$ & $67.0 \pm 8.8$ \\
$\begin{array}{l}\text { Tumor location } \\
\text { Upper }\end{array}$ & $0(0)$ & $1(6.7)$ & $1(20.0)$ \\
Middle & $14(66.7)$ & $9(60.0)$ & $4(80.0)$ \\
Lower & $7(33.3)$ & $5(33.3)$ & $0(0)$
\end{tabular}

Length of the tumor

(mm)

$\begin{array}{llll}\leq 20 & 15(71.4) & 7(46.7) & 0(0) \\ >20 & 6(28.6) & 8(53.3) & 5(100)\end{array}$

Circumferential extension

$$
\begin{aligned}
& <1 / 4 \\
& 1 / 4-2 / 4 \\
& 2 / 4-3 / 4 \\
& \geq 3 / 4
\end{aligned}
$$

\section{7 (33.3)}

$5(33.3)$

$0(0)$

$12(57.2)$

5 (33.3)

$2(40.0)$

$2(9.5)$

5 (33.3)

$3(60.0)$

Endoscopic gross

appearance

\section{Depressed}

Non-depressed

$$
21(100.0)
$$$$
0(0)
$$

Ulceration

$$
\text { Yes }
$$$$
\text { No }
$$

$0(0)$
$21(100.0)$

Time from diagnosis to ESD (days)

$\leq 21$
$>21$

$\begin{array}{lll}15(71.4) & 4(26.7) & 0(0) \\ 6(28.6) & 11(73.3) & 5(100.0)\end{array}$

Histologic findings

Dysplasia

$0(0)$

$0(0)$

Squamous carcinoma

$19(90.5)$

$15(100.0) \quad 5(100.0)$

Depth of invasion

Mucosa

\subsection{5}

0.785

0.252

0.013

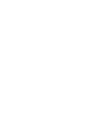

0.100

\begin{tabular}{|c|c|c|c|c|}
\hline \multirow[t]{3}{*}{ Variables } & \multicolumn{3}{|c|}{ Submucosal fibrosis ( $n, \%)$} & \multirow{3}{*}{$\begin{array}{l}P \\
\text { value }\end{array}$} \\
\hline & \multirow{2}{*}{$\begin{array}{l}\text { No } \\
\text { F0 }(N=21) \\
(n, \%)\end{array}$} & \multicolumn{2}{|l|}{ Yes } & \\
\hline & & $\begin{array}{l}\text { F1 (Mild) } \\
(N=15) \\
(n, \%)\end{array}$ & $\begin{array}{l}\text { F2 } \\
\text { (Severe) } \\
(N=5) \\
(n, \%)\end{array}$ & \\
\hline Lymphovascular invasion & & & & 0.583 \\
\hline Absence & $18(94.7)$ & $15(100.0)$ & $5(100.0)$ & \\
\hline Presence & $1(5.3)$ & $0(0)$ & $0(0)$ & \\
\hline
\end{tabular}

Table 1 (continued)

$E S D$ endoscopic submucosal dissection

Table 2 Multivariate logistic regression analysis of factors predicting submucosal fibrosis

\begin{tabular}{llll}
\hline Variables & & HR $(\mathbf{9 5} \% \mathrm{Cl})$ & P value \\
\hline Endoscopic & Depressed & $\begin{array}{l}12.820(2.506- \\
\text { 65.456) }\end{array}$ & 0.002 \\
gross & & Ref & \\
appearance & Non-depressed & $6.056(1.129-$ & 0.036 \\
Length of the & $>20$ & $32.492)$ & \\
tumor (mm) & & Ref & \\
& $\leq 20$ & $10.214(2.021-$ & 0.005 \\
Timing of ESD & $>21$ & $51.630)$ & \\
from initial & & Ref & \\
biopsy (days) & $\leq 21$ &
\end{tabular}

HR hazard ratio, ESD endoscopic submucosal dissection

initial biopsy. In particular, severe submucosal fibrosis (F2) was closely related to larger tumor size $(>20 \mathrm{~mm})$ and delayed ESD after initial biopsy. The multivariate logistic regression analysis indicated that a depressed type tumor (vs. nondepressed type tumor, $P=0.002$ ), length of tumor greater than $20 \mathrm{~mm}$ (vs. length of tumor $\leq 20 \mathrm{~mm}, P=$ 0.036), and delayed ESD after initial biopsy (vs. early ESD after initial biopsy, $P=0.005$ ) were independent factors that predicted endoscopic submucosal fibrosis (Table 2).

\section{Outcome of ESD according to endoscopic submucosal fibrosis}

The overall en bloc resection rate was not different between the lesions accompanied by endoscopic submucosal fibrosis and without endoscopic submucosal fibrosis. However, the severity of endoscopic submucosal fibrosis was significantly associated with higher immediate bleeding rate. Also, one case of perforation displayed an endoscopic submucosal fibrosis of F2. On the other hand, delayed bleeding was not related to the degree of the endoscopic submucosal fibrosis. (Table 3) 
Table 3 Immediate bleeding, delayed bleeding, and perforation according to the degree of endoscopic submucosal fibrosis

\begin{tabular}{lllll}
\hline Variables & \multicolumn{2}{l}{ Endoscopic submucosal fibrosis } & \multirow{2}{*}{ P value } \\
\cline { 2 - 3 } & No (F0) & Mild (F1) & Severe (F2) & \\
\hline Immediate bleeding & & & & 0.018 \\
Yes $(n=34)$ & $14(66.7)$ & $15(100.0)$ & $5(100.0)$ & \\
No $(n=7)$ & $7(33.3)$ & $0(0.0)$ & $0(0.0)$ & \\
Delayed bleeding & & & & 0.284 \\
Yes $(n=3)$ & $2(9.5)$ & $0(0.0)$ & $1(20.0)$ & \\
No $(n=38)$ & $19(90.5)$ & $15(100.0)$ & $4(80.0)$ & \\
Perforation & & & & 0.025 \\
Yes $(n=1)$ & $0(0.0)$ & $0(0.0)$ & $1(20.0)$ & \\
No $(n=40)$ & $21(100.0)$ & $15(100.0)$ & $4(80.0)$ & \\
\hline
\end{tabular}

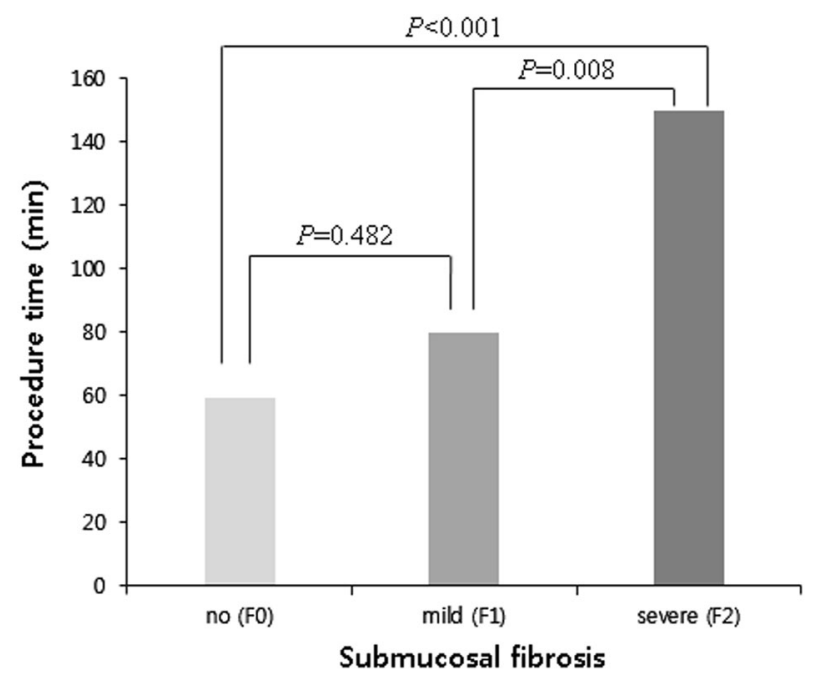

Fig. 3 Relationship between procedure times and degree of endoscopic submucosal fibrosis. F0, no fibrosis; F1, mild fibrosis; F2, severe fibrosis

The mean procedure time was $77.9 \mathrm{~min}$. Procedure time was correlated to the degree of endoscopic submucosal fibrosis (Fig. 3). The mean procedure time of the ESD according to the degree of the endosopic submucosal fibrosis were as follows: F0, 59.4 min (ranges 43.6-75.1 min), F1, 79.8 minutes (ranges 57.4-102.2 min), and F2, $150.0 \mathrm{~min}$ (ranges $60.7-239.3 \mathrm{~min}$ ). Obviously, as the endoscopic submucosal fibrosis became more severe, the required procedure time increased.

\section{Relationship between endoscopic and histologic estimates of submucosal fibrosis}

The comparison of endoscopic and histologic grading of submucosal fibrosis is provided in Table 4. The
Table 4 Degree of endoscopic submucosal fibrosis in relationship to histologic submucosal fibrosis

\begin{tabular}{llll}
\multirow{2}{*}{ Endoscopic submucosal fibrosis } & \multicolumn{3}{l}{ Histologic submucosal fibrosis } \\
\cline { 2 - 4 } & No (F0) & Mild (F1) & Severe (F2) \\
\hline No (F0) & 11 & 2 & 0 \\
Mild (F1) & 0 & 13 & 2 \\
Severe (F2) & 0 & 0 & 5 \\
\hline
\end{tabular}

$K=0.808(p<0.001)$

concordance was $87.9 \%$ (Kendall's tau-b and $k$ coefficients were 0.877 and 0.808 , respectively) $(P<0.001)$.

\section{Discussion}

In this study, three predictive factors of endoscopic submucosal fibrosis were identified: depressed type tumor, length of tumor greater than $20 \mathrm{~mm}$, and delayed ESD after initial biopsy. These findings indicate that technical difficulty will be encountered during esophageal ESD for such lesions. This study also demonstrated that the extent of the endoscopic submucosal fibrosis is related to the frequency of immediate bleeding and endoscopic submucosal fibrosis was classified as F2 in a case with perforation. In addition, as the endoscopic submucosal fibrosis became more severe, the procedure time took longer.

It has been reported that as the size of lesions increased, the dangers of bleeding, perforation, incomplete resection, and delays in procedure increased ${ }^{14-17}$. Furthermore, some studies reported that endoscopic submucosal fibrosis was closely related to the size of tumor and endoscopic gross appearance ${ }^{10,11}$. However, there have been no examinations of the relationship between the degree of submucosal fibrosis and cliniopathological factors in SSEN. Similar to previous studies on gastric tumors, the present study demonstrated that a length of tumor greater than $20 \mathrm{~mm}$ and endoscopic depressed type were independent risk factors for endoscopic submucosal fibrosis. Therefore, we suggest that prior to perform esophageal ESD, a cautious approach is necessary when the length of tumor is over $20 \mathrm{~mm}$ and depressed endoscopically since the possibility of endoscopic submucosal fibrosis is very high.

The optimal timing from initial biopsy to ESD remains undefined. A previous study suggested an endoscopic resection should be performed within 21 days after a biopsy because interval over this period may be associated with non-lifting signs in endoscopically-resectable colorectal cancer ${ }^{18}$. Our results also demonstrated that delayed ESD over 21 days after initial biopsy was a significant risk factor of submucosal fibrosis, which suggests that a longer interval between diagnostic biopsies and ESD may lead to 
submucosal fibrosis. It is plausible that a biopsy can induce mucosal ulceration and cause scarring to change over time, which in turn might lead to submucosal fibrosis. Immediate ESD within 21 days after initial diagnostic biopsy should be considered before submucosal fibrosis proceeds.

We observed that the more severe the endoscopic submucosal fibrosis is, the longer the procedure time takes. Several previous studies also have shown that submucosal fibrosis could be recognized as a predictor of prolonged

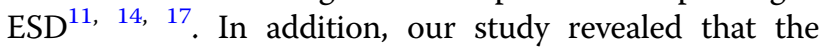
severity of the endoscopic submucosal fibrosis is associated with the frequency of immediate bleeding and perforation. The prolonged duration of the procedure and high frequency of complications are due to the technical difficulty of dissecting deeper tissue for complete resection where fibrosis occurs. Therefore, when submucosal fibrosis is detected, it is necessary to perform ESD more carefully.

We tested the concordance between the endoscopic and histologic classifications of submucosal fibrosis. The endoscopic classification directly reflects the histological classification of submucosal fibrosis. This suggests that the endoscopic classification could predict the ESD outcome with a high degree of concordance. The endoscopic assessment of submucosal fibrosis is an objective measure of submucosal fibrosis.

Our study has some limitations. First, this study was based on a small sample size. Though not statistically significant, our results showed that endoscopic submucosal fibrosis has the tendency to be associated with the depth of invasion $(p=0.063)$. Further studies with large sample size might lead to statistical significance of the values between the groups. Second, because there is no objective definition of endoscopic submucosal fibrosis and histologic submucosal fibrosis, a subjective classification of a submucosal fibrosis from the observer's point of view may have caused a difference from other previous studies. To overcome this limitation, we tested the concordance between the endoscopic and histologic classifications of submucosal fibrosis, which demonstrated strong agreement $(\kappa=0.808, p<0.001)$. Third, there might be a bias due to the retrospective nature of the current study. Finally, additional endoscopic predictive factors, including the expertize of the endoscopist and the method of ESD were not included in this study. Thus, further large prospective studies are needed to validate our study. To our knowledge, however, our study is the first to describe the relationship between the degree of endoscopic submucosal fibrosis and the outcome of ESD in SSEN. In addition, the prediction of endoscopic submucsoal fibrosis prior to ESD would be beneficial for safe and complete endoscopic treatment of SSEN. Therefore, our study provides an insight into the validation of the predictors of endoscopic submucosal fibrosis prior to esophageal ESD.

In conclusion, we found that the endoscopic submucosal fibrosis of SSEN was closely associated with length of tumor, endoscopic depressed type, and timing of ESD from initial biopsy. The endoscopists need to apply extra caution when the length of tumor is over $20 \mathrm{~mm}$, depressed, or over 21 days past after initial biopsy. Esophageal ESD for SSEN should be attempted immediately after biopsy.

\section{Study Highlights}

\section{What is current knowledge}

- Endoscopic submucosal dissection (ESD) is an effective treatment modality for superficial squamous esophageal neoplasia (SSEN)

- Submucosal fibrosis is an important obstacle to successful ESD

\section{What is new here}

- Length of tumor greater than $20 \mathrm{~mm}$ and endoscopic depressed type are endoscopic predictive factors of submucosal fibrosis in SSEN

- To avoid submucosal fibrosis, ESD should be attempted immediately after biopsy for the diagnosis of SSEN

\section{Translational impact}

- The endoscopists need to apply extra caution when the length of tumor is over $20 \mathrm{~mm}$, depressed, or over 21 days past after Initial biopsy.

- Esophageal ESD for SSEN should be attempted immediately after biopsy.

\section{Author details}

${ }^{1}$ Division of Gastroenterology, Department of Internal Medicine, Incheon St. Mary's Hospital, College of Medicine, The Catholic University of Korea, Incheon, Korea. ${ }^{2}$ Division of Gastroenterology, Department of Internal Medicine, Seoul St. Mary's Hospital, College of Medicine, The Catholic University of Korea, Seoul, Korea. ${ }^{3}$ Department of Pathology, Incheon St. Mary's Hospital, College of Medicine, The Catholic University of Korea, Incheon, Korea

\section{Conflict of interest}

Guarantor of the article: Byung-Wook Kim, MD, PhD.

Specific author contributions: B.-W.K. designed this study. B.-W.K., B.-I.L., and C.-H.L. performed the procedure and gathered data. J.K. reviewed and interpreted pathologic results. H.H.L. and J.S.K. managed the data. C.-W.H. managed the data, performed statistical analysis, and wrote the manuscript. Financial support: None.

Potential competing interests: None.

Received: 1 December 2017 Revised: 27 February 2018 Accepted: 10 April 2018

Published online: 15 June 2018 


\section{References}

1. Odagiri, $H$. et al. Hospital volume and adverse events following esophageal endoscopic submucosal dissection in Japan. Endoscopy 49, 321-326 (2017).

2. Ning, B., Abdelfatah, M. M. \& Othman, M. O. Endoscopic submucosal dissection and endoscopic mucosal resection for early stage esophageal cancer. Ann. Cardiothorac. Surg. 6, 88-98 (2017).

3. Nagami, Y. et al. The five-year survival rate after endoscopic submucosal dissection for superficial esophageal squamous cell neoplasia. Dig. Liver. Dis. 49, 427-433 (2017).

4. Kim, J. S., Kim, B. W. \& Shin, I. S. Efficacy and safety of endoscopic submucosa dissection for superficial squamous esophageal neoplasia: a meta-analysis. Dig. Dis. Sci. 59, 1862-1869 (2014).

5. Yamashina, T. et al. Long-term outcome and metastatic risk after endoscopic resection of superficial esophageal squamous cell carcinoma. Am. J. Gastroenterol. 108, 544-551 (2013).

6. Toyonaga, T. et al. 1,635 Endoscopic submucosal dissection cases in the esophagus, stomach, and colorectum: complication rates and long-term outcomes. Surg. Endosc. 27, 1000-1008 (2013).

7. Ono, S. et al. Long-term outcomes of endoscopic submucosal dissection for superficial esophageal squamous cell neoplasms. Gastrointest. Endosc. 70, 860-866 (2009)

8. Ishihara, R. et al. Local recurrence of large squamous-cell carcinoma of the esophagus after endoscopic resection. Gastrointest. Endosc. 67, 799-804 (2008).

9. Tsujii, Y. et al. Clinical outcomes of endoscopic submucosal dissection for superficial esophageal neoplasms: a multicenter retrospective cohort study. Endoscopy 47, 775-783 (2015).
10. Higashimaya, M. et al. Outcome of endoscopic submucosal dissection for gastric neoplasm in relationship to endoscopic classification of submucosal fibrosis. Gastric Cancer 16, 404-410 (2013).

11. Jeong, J. Y. et al. Does submucosal fibrosis affect the results of endoscopic submucosal dissection of early gastric tumors? Gastrointest. Endosc. 76, 59-66 (2012).

12. Matsumoto, A. et al. Outcome of endoscopic submucosal dissection for colorectal tumors accompanied by fibrosis. Scand. J. Gastroenterol. 45, 1329-1337 (2010).

13. Endoscopic Classification Review Group. Update on the paris classification of superficial neoplastic lesions in the digestive tract. Endoscopy 37, 570-578 (2005).

14. Ahn, J. Y. et al. Procedure time of endoscopic submucosal dissection according to the size and location of early gastric cancers: analysis of 916 dissections performed by 4 experts. Gastrointest. Endosc. 73, 911-916 (2011).

15. Mannen, K. et al. Risk factors for complications of endoscopic submucosal dissection in gastric tumors: analysis of 478 lesions. J. Gastroenterol. 45, 30-36 (2010).

16. Lee, T. H. et al. Appropriate indications for endoscopic submucosal dissection of early gastric cancer according to tumor size and histologic type. Gastrointest. Endosc. 71, 920-926 (2010).

17. Chung, I. K. et al. Therapeutic outcomes in 1000 cases of endoscopic submucosal dissection for early gastric neoplasms: Korean ESD Study Group multicenter study. Gastrointest. Endosc. 69, 1228-1235 (2009).

18. Han, K. S. et al. Prolongation of the period between biopsy and EMR can influence the nonlifting sign in endoscopically resectable colorectal cancers. Gastrointest. Endosc. 67, 97-102 (2008). 